\title{
Incidence of Aspiration Pneumonia in Patients with Altered Consciousness Admitted in DMCH
}

MD. RASHED ALAM CHOWDHURY, ${ }^{1}$ KHAN ABUL KALAM AZAD, ${ }^{2}$ MAHMUDUR RAHMAN SIDDIQUI, ${ }^{3}$ SANJEEDA SAAD 4

\begin{abstract}
Background: Aspiration Pneumonia is a common complication in patients with altered consciousness if general supportive care is not taken properly. There is no national study on incidence of aspiration pneumonia in hospitalized patients with altered consciousness. This study was done to see the incidence of aspiration pneumonia in patients with altered consciousness admitted in a tertiary care hospital in our country.
\end{abstract}

Methods: This was a prospective observational study conducted among the 50 adult patients of aspiration pneumonia with altered consciousness admitted in the medicine department of $\mathrm{DMCH}$, during January 2010 to December 2010. Aspiration pneumonia was confirmed by clinical examination and laboratory investigations. Assessment of altered conscious patient was done by application of the Glasgow Coma Scale. Case record forms with appropriate questionnaire were filled for all patients.

Results: The mean $( \pm S D)$ age was $38.9 \pm$ I 7.3 years. Maximum numbers $(24.5 \%)$ of patients were found in the age group of 5 I-60 years. Male female ratio was 3:I. Fever, chest pain and productive cough were the most common clinical features. Stroke (64\%), Encephalitis (16\%) and Hepatic encephalopathy (16\%) were found as main precipitating factors.

Key words: Aspiration Pneumonia, Altered consciousness.

\section{Introduction:}

Aspiration pneumonia is the second most frequent principal diagnosis among the hospitalized patients with altered consciousness. In United States several studies indicate that $5-15 \%$ of community acquired pneumonia results from aspiration pneumonia. Approximately $10 \%$ of patients who are hospitalized due to altered consciousness or drug overdose will have aspiration pneumonia. ${ }^{1}$ Aspiration pneumonia is more common in extremely young and old patients and in males than females. ${ }^{2}$ The mortality rate of aspiration pneumonia is approximately $1 \%$ in outpatient setting and upto $25 \%$ in those requiring hospitalization. The mortality rates for severe chemical pneumonitis can be upto $70 \%{ }^{3}$

Aspiration pneumonia refers to the pulmonary consequences (inflammation of lung parenchyma and bronchial tubes) resulting from the abnormal entry of fluid, particulate exogenous substances, endogenous secretions or gastric contents into the lower airways. Acute inflammatory phase

1. Assistant Professor, Dept. of Medicine, Jahurul Islam Medical College Hospital, Bajitpur, Kisoregonj.

2. Professor, Dept. of Medicine, Dhaka Medical College Hospital, Dhaka.

3. Assistant Professor, Dept. of Medicine, Anwer Khan Modern Medical College Hospital, Dhaka.

4. Post Graduate Student, Dept. of Surgery, BSMMU, Dhaka. Correspondence: Dr. Md. Rashed Alam Chowdhury, Assistant Professor, Dept. of Medicine, Jahurul Islam Medical College Hospital, Bajitpur, Kisoregonj. response involves massive recruitments of neutrophil with systemic elaboration of various cytokine (mainly IL-8) mediated cascades. However, aspiration may causes $-\mathbf{a}$. Chemical pneumonitis: aspiration of gastric juice may cause fatal damage (Mendelson's Syndrome) including hemorrhagic tracheobronchitis, pulmonary edema and ARDS. b. Bacterial infection: infection is typically caused by the aspiration of oral flora especially in patients with poor oropharyngeal hygiene. The pathogens that commonly produce aspiration pneumonia are Streptococcus pneumoniae, Haemophilus influenzae, gram-negative bacilli, Staphylococcus aureus and anaerobes. Aspirated materials in lungs may lead to abscess, empyema, bronchiectasis and acute respiratory failure. ${ }^{4}$ Clinical features of aspiration pneumonia involving anaerobic bacteria include: Indolent symptoms, usually compromised consciousness, absence of rigor, failure to recover, sputum often has a putrid odor. ${ }^{\mathbf{5}} \mathbf{c}$. Obstruction: Large volume of aspirated materials may lead to acute airway obstruction with lobar or segmental atelectasis. ${ }^{4}$ Complications following aspiration are presumably related to the frequency, volume, character of the aspirates, underlying diseases and level of consciousness. ${ }^{6}$ Predisposing factors of aspiration pneumonia: Main predisposing factors are- Impaired consciousness (acute stroke, seizures, ICSOL, drug or alcohol overdose, hepatic encephalopathy, head injury, hypoglycemia etc.), Swallowing disorders (bulbar palsy, acute stroke, multiple sclerosis, Parkinson's disease, GBS, 
myasthenia gravis.) and Others (N-G tube feeding, tracheostomy, protracted vomiting, recumbent position, GERD). ${ }^{2}$

On the other hand, level of consciousness is the relative state of awareness of self and the environment and ranges from fully awake to comatose state. ${ }^{7}$ Systematic assessment of an altered conscious patient can be done by the application of the Glasgow Coma scale (minimum score- 3, maximum score- 15$){ }^{8}$

Internationally aspiration pneumonia is considered as a common disease but no recent statistics are available. Aspiration pneumonia in altered conscious patient is not uncommon in Bangladesh. In Bangladesh there is no exact incidence of aspiration pneumonia. This study is an attempt to identify the incidence of Aspiration pneumonia in hospitalized patients with altered consciousness. This information is not currently available and is expected to create a baseline of information for future reference.

\section{Method and Material:}

This Prospective Observational study was done in the department of Medicine, of Dhaka Medical College Hospital during the period between January-2010 to December-2010. 50 adult hospitalized patients with altered consciousness in the department of Medicine of Dhaka Medical College Hospital were enrolled in this prospective study. Informed written consent was obtained from the patient's attendant after full explanation of the disease process. After admission in the indoor, patients with altered consciousness seen by unit doctor were screened by study physician. Evaluation was made by history and physical examination in a structured case record form (CRF) by the study physician. Patients diagnosed clinically as aspiration pneumonia was screened in the study. Assessment of altered conscious patient was done by application of the Glasgow Coma Scale. Hematologic measurements (TC of WBC, $\mathrm{Hb} \%$, ESR, platelet count), chest X-ray, sputum for Gram staining and culture sensitivity, blood gas analysis, blood culture, blood urea, creatinine and random blood sugar were done. For scanty production of cough, patient's sputum was collected after nebulization by hypertonic normal saline. Other investigations such as CT brain, CSF Study, LFT were done to find out the cause of altered consciousness. Categorical data was presented as frequency and percentage and continuous variable presented as mean and standard deviation. All data were processed and analyzed manually and by SPSS (Statistical Package for Social Science) 16 windows version.

\section{Result:}

This prospectively documented study enrolled 50 cases of altered conscious patients. The mean $( \pm \mathrm{SD})$ age was 57.42 \pm 13.63 years with ranged from 25 to 90 years and maximum number $(30 \%)$ of patients was found in the age group of 51 60 years. Out of 50 patients, $35(70 \%)$ patients were male and $15(30 \%)$ patients were female with a ratio of $2.33: 1.26 \%$ of the study patients developed aspiration pneumonia in whom $16 \%$ are male and $10 \%$ are female. Fever, cough and respiratory distress were present in most of the patients who developed aspiration pneumonia among the study patients. During physical examination about $20 \%$ of the patients had $100^{0}-102^{\circ} \mathrm{F}$ temperature, $36 \%$ patients had consolidation in chest $\mathrm{x}$-ray and all patients had altered mental status (GCS was $7-12)$. The mean $( \pm \mathrm{SD})$ respiratory rate was $25.14 .0 \pm 7.47 /$ minute with range from 14 to $40 /$ minute. The main precipitating factors causing altered consciousness of the study patients were stroke, encephalitis and encephalopathy due to hepatic encephalopathy and uremia.

Table-I

Age distribution of the study patients $(n=50)$.

\begin{tabular}{lcc}
\hline Age in years & Number of patients & Percentage \\
\hline $25-40$ & 06 & 12 \\
$41-50$ & 10 & 20 \\
$51-60$ & 15 & 30 \\
$61-70$ & 13 & 26 \\
$71-80$ & 04 & 08 \\
$81-90$ & 02 & 04 \\
\hline
\end{tabular}

Mean \pm SD $=57.42 \pm 13.63$

Range $(\min -\max )=(25-90)$

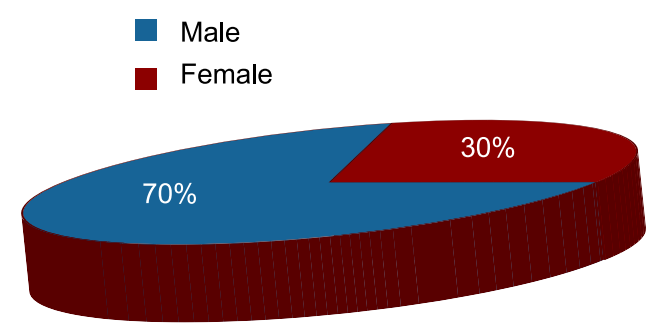

Fig.-1: Distribution of patients according to $\operatorname{sex}(n=50)$.

Table II

Distribution of the patients developing Aspiration Pneumonia among the study patients $(n=13)$.

\begin{tabular}{lccc}
\hline Sex & $\begin{array}{c}\text { Number of } \\
\text { patients }(\mathrm{n})\end{array}$ & Percentage & $\begin{array}{c}\text { Total } \\
\text { Percentage }\end{array}$ \\
\hline Male & 08 & 16 & 26 \\
Female & 05 & 10 & \\
\hline
\end{tabular}


Table III

Clinical features of aspiration pneumonia among the study patients $(n=13)$.

\begin{tabular}{lcc}
\hline Clinical feature & Number of patients & Percentage \\
\hline Fever & 13 & 100 \\
Cough & 12 & 92.3 \\
Respiratory distress & 13 & 100 \\
Vomiting & 05 & 38.5 \\
Chest pain & 06 & 46.2 \\
\hline
\end{tabular}

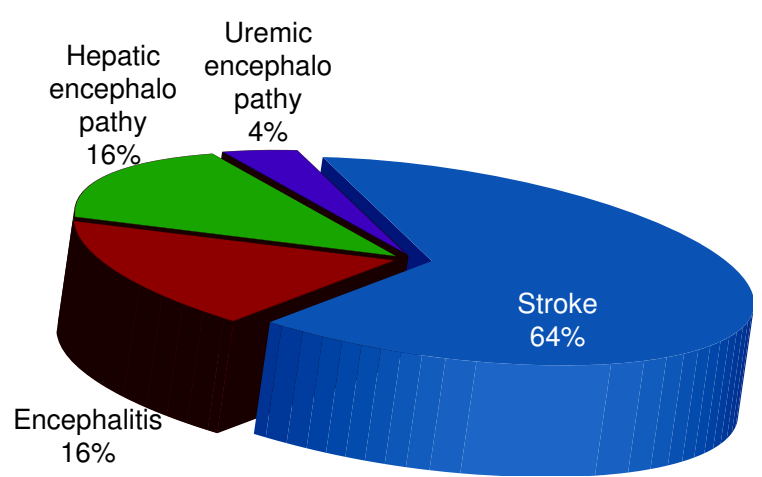

Fig.-2: Frequency of precipitating factors among the study patients $(n=50)$.

\section{Discussion:}

In this small series of patients with altered consciousness in a tertiary level hospital, aspiration pneumonia could be identified in $26 \%$ (13) of patients. Out of $13,61.5 \%$ (8) were male and $38.5 \%(5)$ were female. Internationally, approximately $10 \%$ of patients who are hospitalized due to altered consciousness will have aspiration pneumonia. Several studies showed that 5-15\% of community acquired pneumonia results from aspiration pneumonia. So, it can be seen that in this study incidence of aspiration pneumonia in altered conscious hospitalized patients is slightly higher than other study which was done internationally previously. The cause of higher incidence may be due to crowded patients in small area, malnutrition of patients, lack of knowledge of patients' attendant and health care provider regarding prevention of aspiration pneumonia and care of patients, poverty and small number of doctor $\&$ nurses in relation to large number of patients.

The mean $( \pm \mathrm{SD})$ age was $57.42 \pm 13.63$ years with ranged from 25 to 90 years and maximum number $(30 \%)$ of patients was found in the age group of $51-60$ years. El-Solh ${ }^{1}$ found that the mean $( \pm \mathrm{SD})$ age was $80.2 \pm 6.5$ years in their study. In this study it was found that $70 \%$ (35) of the patients were male and $30 \%(15)$ were female. Male female ratio was 2.33:1. El-
Solh $^{1}$ found that out of 69 patients 43 were males and 26 were females. In present study all the patients had altered consciousness. GCS was ranging from 7 to 12 in all study patients. Fever, cough and respiratory distress were present in most of the patients who developed aspiration pneumonia among the study patients which is similar to little previous study. Few patients developed chest pain and vomiting. Temperature was $100-102^{\circ} \mathrm{F}$ in most of the patients; few patients had more than $103^{\circ} \mathrm{F}$ temperature. Mean oxygen saturation $\left(\mathrm{SpO}_{2}\right)$ was $94.3 \%$ ranging from $85 \%-100 \%$. Mean $( \pm \mathrm{SD})$ respiratory rate was $25.14 \pm 7.47 / \mathrm{min}$ ranging from 14 40/min. On physical examination, raised temperature, tachypnea, hypoxia, crepitations and rhonchi were the main findings in patients with aspiration pneumonia. Few patients developed hypotension and cyanosis. Bynum and Pierce (1976) found fever, cough, respiratory distress, tachypnea, diffuse rales, and serious hypoxemia, cyanosis, wheezing, and apnea in approximately one third of the cases $(n=50)$ in their study. ${ }^{9}$ Elsolh and Pietrantoni (2003) found dyspnea, fever, and delirium in the majority of patients in their study. ${ }^{1}$ In present study all the patients had reduced consciousness. Stroke was found in $32(64 \%)$ patients, encephalitis was found in $8(16 \%)$ patients, hepatic encephalopathy was found in 8 (16\%) patients and 2 patients had uremic encephalopathy. In this study it revealed that neurological disorder was the main precipitating factor for developing aspiration pneumonia in altered conscious patients. Finegold (1991) ${ }^{10}$ found that the main factors which predispose to aspiration pneumonia include a reduced level of consciousness, dysphagia, periodontal disease, and mechanical interference that is related to the insertion of various tubes into the respiratory or gastrointestinal tracts. ${ }^{10} \mathrm{El}$ - Solh $(2003)^{1}$ found that $78 \%$ patients had stroke and $35 \%$ patients had COPD in their study.

This descriptive study is a study with small sample and requires further studies from different levels of hospitals. A nationally representative surveillance system for Aspiration pneumonia could replace periodic small studies.

\section{Conclusion:}

This prospective observational study was done in aspiration pneumonia of hospitalized patients with altered consciousness. $26 \%$ of hospitalized patients with altered consciousness developed aspiration pneumonia. Incidence of aspiration pneumonia in altered conscious hospitalized patients is slightly higher than other study which was done internationally previously. The cause of higher incidence may be due to crowded patients in small area, malnutrition of patients, lack of knowledge of patient's attendant and health care provider regarding prevention of aspiration pneumonia 
and care of patients, poverty and small number of doctor \& nurses in relation to large number of patients. So, education of patient's attendant and health care provider, proper general and supportive care of patients, alertness and increase number of doctors can reduce incidence of aspiration pneumonia in altered conscious hospitalized patients.

\section{Conflict of Interest: None.}

\section{References:}

1. El-Solh AA, Pietrantoni C, Bhat A, Aquilina AT, Okada M, Grover V, et al. Microbiology of severe aspiration pneumonia in institutionalized elderly. Am J Respir Crit Care Med 2003; 167(12): 1650-4.

2. Marik PE. Aspiration pneumonitis and aspiration pneumonia. N Engl J Med 2001; 344(9): 665-71.

3. DeLegge MH. Aspiration pneumonia: incidence, mortality, and at-risk populations. J Parenter Enteral Nutr 2002; 26(6 suppl): S19-S25.
4. Adnet F, Baud F. Relation between Glasgow Coma Scale and aspiration pneumonia. Lancet 1996; 348(9020): 123-4.

5. Bartlett JG. Anaerobic bacterial pneumonitis. Am Rev Respir Dis 1979; 119(1): 19.

6. Bartlett JG, Gorbach S. The triple threat of aspiration pneumonia. Chest 1975; 68(4): 560-6.

7. Fauci AS, Braunwald E, Kasper DL, Hauser SL, Longo DL, Jameson JL, et al, editors. Harrison's Principles of Internal Medicine: $.17^{\text {th }}$ ed. 2008. p. 2486.

8. Allen CMC, Lueck CJ, Dennis M. Neurological disease. In: Colledge NR, Walker BR, Ralston SH, editors. Davidson's Principles and Practice of Medicine. 21st ed. Edinburgh: Churchill Livingstone/Elsevier; 2010. p. 1131-235.

9. Bynum LJ, Pierce AK. Pulmonary aspiration of gastric contents. Am Rev Respir Dis 1976; 114(6): 1129.

10. Finegold SM. Aspiration pneumonia. Rev Infect Dis 1991; 13(Supplement 9): S737-S42. 